\title{
Design and implementation of intelligent irrigation system
}

\author{
Xiaofei Hu, Xia Sun*, Qinghong Li, Qianqian He, and Yajun Li \\ School of Electrical and Information Engineering, Anhui University of Science and Technology, \\ Huainan 232001, China,
}

\begin{abstract}
In view of the problems existing in traditional irrigation, such as high time cost, poor reliability, waste of water resources. The intelligent irrigation system based on STM32 and BC95 is designed and implemented. The soil information is received through temperature sensor and humidity sensor, which is sent from the sampling node to the remote terminal serial port. The controller sends the signal to the output end for intelligent irrigation. The practice shows that the wireless communication mode of data transmission using STM32 and NB-IoT (narrow band-internet of things) technology can meet the requirements of reducing the time cost and enhancing the reliability of the system, and can meet the goal of data transmission of intelligent irrigation system and water-saving irrigation. it can be seen that the soil moisture data in the figure significantly changes.
\end{abstract}

Keywords: Intelligent irrigation; STM32F103 chips; NB - IoT (narrow band-internet of things) technology; Cloud platform technology; BC95.

\section{Introduction}

Due to the uneven distribution of water resources and the increasing demand for fresh water resources, the shortage of fresh water resources will become a serious problem faced by all countries in the world in the near future. The proportion of agricultural water in fresh water consumption is relatively high in China. ${ }^{[1]}$ At present, water-saving irrigation in China is still in its infancy. Therefore, in view of this situation, an intelligent irrigation system with lower power consumption, lower cost and wider application scenarios is designed.

Aiming at the problems existing in traditional irrigation, intelligent irrigation is proposed. In order to solve the previous bluetooth, ZigBee, WiFi, etc in intelligent irrigation the disadvantage of short distance wireless communication technology, and GPRS or $4 \mathrm{~g}$ module on the network capacity is insufficient, the narrowband Internet of things technology combined with agriculture, not only can well adapt to the wild environment of information transmission, still can save fresh water resources, to a certain extent, improve the agricultural production. Therefore, it is necessary to apply NB-IoT ${ }^{[2]}$ technology in agricultural irrigation to make agricultural irrigation more intelligent, energy saving and scientific.

\footnotetext{
*Corresponding author: xiasun@aust.edu.cn
} 
The hardware terminal of intelligent irrigation system based on NB-IOT mainly includes its main control module and peripheral circuit, control module circuit, USB level conversion circuit, power supply circuit, NB wireless transmission module and peripheral circuit and sensor data acquisition circuit. ${ }^{[3]}$ The intelligent irrigation system based on NBIoT can collect soil temperature, humidity and light intensity, and then decide whether to turn on or off irrigation based on the collected information. The terminal node adopts soil moisture sensor and air temperature and humidity sensor to collect environmental parameters, and transmit the parameters to the master chip, and the master chip will transmit the data to the NB module through serial communication.

The NB module is responsible for uploading data to the cloud. At the same time, NB module is also responsible for receiving the downward control instructions of the upper computer control management platform, and then sending the instructions down to the irrigation control module through the main control module. NB transmission node is the key to the data transmission in the whole system. Its function is to transmit the data collected by sensors to the telecom cloud platform through the core network in the form of binary code stream, and finally to the management platform. The upper wingneng management platform is responsible for data query and management, and the upper computer control terminal makes the final irrigation decision, and then sends the irrigation instruction to the terminal node to control whether to choose irrigation. In addition, the interactive interface can display the real-time monitoring data and the dynamic curve of the sensor data to the monitoring personnel and complete other functions of the system. ${ }^{[4]}$

\section{The overall structure of the hardware system}

NB-IoT intelligent irrigation hardware terminal is mainly composed of master control module and peripheral circuit, control module circuit, USB level conversion circuit, power supply circuit, NB wireless transmission module and peripheral circuit, sensor data acquisition circuit. The overall architecture of the system hardware terminal is shown in Figure 1.

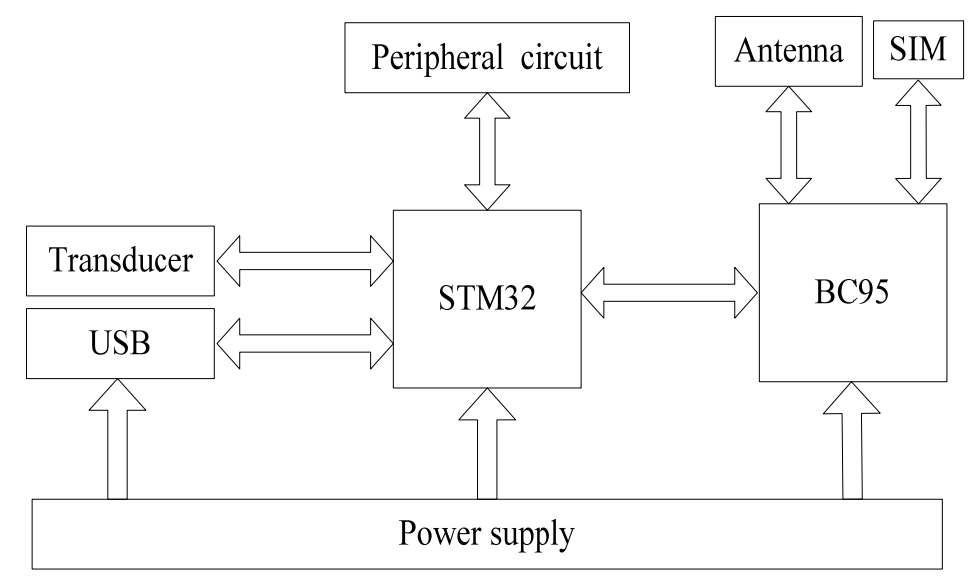

Fig. 1. Overall architecture of hardware terminal.

The main control module uses STM32F103 as the main control chip. the SIM card is supplied by the BC95-B5, and the power supply of the control module is provided by the high and low level of the main control chip. USB level conversion circuit. USB level conversion circuit is used to communicate between MCU and PC. ${ }^{[5-6]}$ NB-IoT module and peripheral circuits include BC95-B5 module, antenna and SIM card interface circuit, etc. The sensor data acquisition circuit is responsible for collecting intelligent irrigation 
parameter data, including soil moisture and air temperature and humidity acquisition circuit. The control module adopts the relay as the control module, and the main control chip is connected through the serial port, and the control signal of the main control chip is used to control its on-off. Due to the limited experimental conditions, the control module is not really connected to the pump, but the LED indicator is on or off to simulate the pumping state.

NB-IoT module and peripheral circuits include NB-IoT module, interface circuit, ${ }^{[7]}$ SIM card circuit, etc. BC95 is a NB-IoT wireless communication module produced by Shiyuan Communications, which first passed the national mandatory product certification. BC95 is a high performance, low power NB-IoT wireless communication module. BC95 adopts the LCC package that is easier to weld, and can realize the rapid production of modules through the standard SMT equipment, providing customers with a reliable connection method, especially suitable for automated, large-scale, low-cost modern production method. ${ }^{[8]}$

NB-IoT module provides sending and receiving serial ports, so STM32F103 and NBIoT module can use USART serial port communication, STM32F103 has a total of three USART1, USART2 and USART3, respectively. USART is a universal synchronous asynchronous transceiver and UART is a serial asynchronous transceiver. The difference between UART and USART is that USART has one more clock pin. Because of this clock line, the serial port data receiving and receiving do not interfere with each other. USART can also become asynchronous without using this clock line.

\section{Soil moisture acquisition module and temperature and humidity acquisition module}

Temperature affects not only photosynthesis, but also water absorption of plants. Humidity will affect plant transpiration, and too high air humidity will make crops more susceptible to pests and diseases, so the change of temperature and humidity plays a very important role in the growth of plants. Figure 2 is the wiring circuit diagram of DHT11 and STM32F103 modules

DHT11

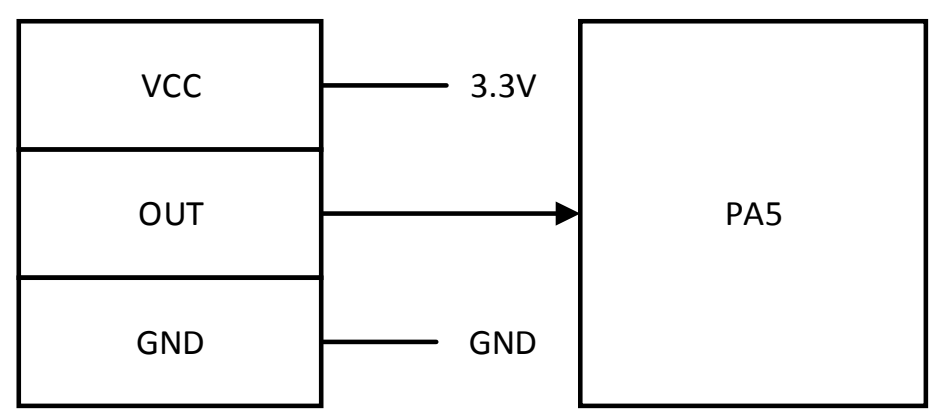

Fig. 2. Pin connection between temperature and humidity acquisition module and main control module.

The VCC pin of DHT11 is the power supply, and the power supply voltage is $3.3 \mathrm{~V}$. GND pin is grounded. Data is a single-wire serial interface, which is connected with PA5 pin of STM32F103.

NB-IoT module operates mainly through AT instructions. The AT instruction sending program between STM32 and NB-IoT module is the basis of the whole data transmission 
process. The process of registering and entering the network of NB-IoT equipment and data sending using COAP protocol are all based on AT instruction sending program.

At instruction sender process: enter the AT instruction sender first initialize the cache, receive data cache Recedata clear 0 , cache index LEN set to 0. USART1 forwards instructions to PC to facilitate debugging by developers. The serial port USART2 sends AT instruction ATCOM to the NB-IOT module. Enable USART2 data reception interrupt to receive response data from the NB-IoT module. When data is received, it enters the receive callback function. The single-byte data cache, Arecebuf, receives one byte of data. Because STM32 terminal adopts HAL library development, the receiving interrupt function in HAL library can only receive fixed length data each time, so the system adopts the method of single byte receiving data interrupt to realize receiving variable length data. Receiving data cache ReceData values from the single-byte cache. The cache index of Recedata, the receiving data cache, is increased by 1 to store the value in the next single-byte cache. Whether the data receiving has been completed or not, if not, enable interrupt to enter Step 5; If the receive is complete, the receive interrupt is turned off into the following procedure. Delay for a period of time to ensure that the serial port USART2 fully receives data. The serial port USART1 forwards the received response data to the serial port assistant on the $\mathrm{PC}$ for data display. Determines whether the received response contains the expected value, and returns 1 if it does, or 0 if it does not.

First, take the FC28 soil moisture sensor as an example. After the terminal monitoring node is powered on, the collected information changes accordingly by changing the soil moisture, indicating that the terminal node is successful in collecting information. After wiping the surface of the soil moisture sensor with water, it can be seen that the soil moisture data in the figure significantly changes. Temperature and humidity sensors are placed in different environments, and the measured data are shown in Figure 3.

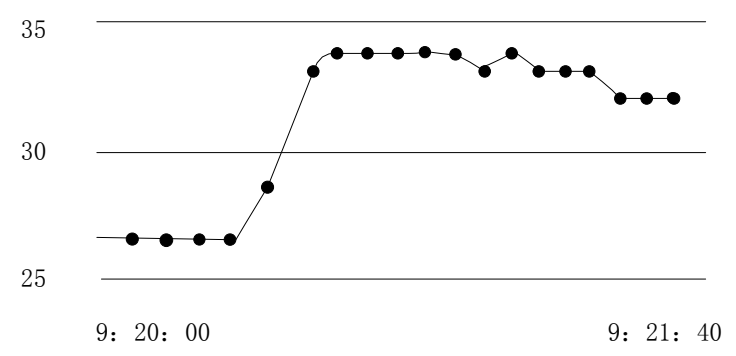

(a) Temperature change

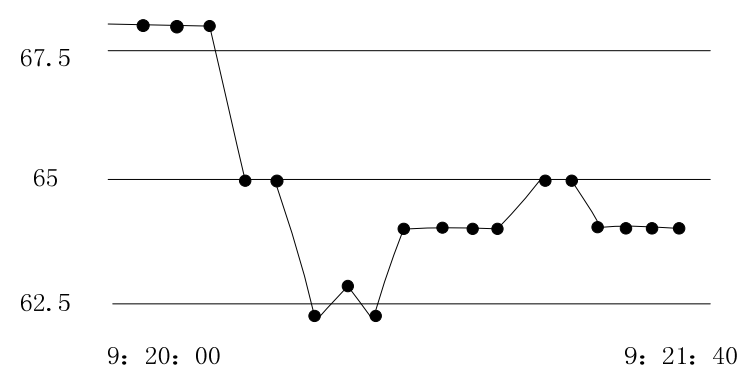

(b) Humidity change

Fig. 3. Test results of temperature and humidity sensor. 


\section{Conclusion}

In view of the intelligent irrigation system, the overall architecture of the hardware terminal of the intelligent irrigation system is mainly designed. The main control module of STM32F103 and its peripheral circuit, power supply management circuit, control module circuit, NB-IOT module, peripheral circuit and sensor circuit are designed and made respectively. In view of the software design and realization of NB-IoT intelligent irrigation system, the design includes the program design and realization of STM32, the NB-IoT module and the server access to the telecom cloud platform for configuration, and the upper computer monitoring platform of intelligent irrigation system. The results show that the intelligent irrigation system can effectively control the irrigation amount, improve the irrigation efficiency and reduce the irrigation time.

Using STM32,sensors, NB - IoT, cloud platform technology such as modern technology and equipment to complete information such as weather, soil moisture of farmland acquisition and wireless transmission, through the intelligent management platform for calculation, data management, and according to the set of irrigation threshold, to decide whether to open irrigation, finally realizes the intelligent of farmland irrigation, so as to improve the scientific nature and the degree of automation of agricultural irrigation. The system can realize remote monitoring of farmland irrigation and realize manual or automatic intelligent irrigation according to the choice of the staff, which improves the utilization rate of agricultural irrigation water and enables the crops to achieve a more suitable growth environment.

\section{Acknowledgements}

This work is supported by Natural Science Research Project of Anhui Province (GXFXZD2016071) Anhui University College Students Innovation and Entrepreneurship Project (S202010361111).

\section{References}

1. Vyacheslav Begishev, Vitaly Petrov, Andrey Samuylov, Dmitri Moltchanov, Sergey Andreev, Yevgeni Koucheryavy, Konstantin Samouylov. Resource allocation and sharing for heterogeneous data collection over conventional 3GPP LTE and emerging NB-IoT technologies[J]. Computer Communications,2018,120.

2. Jerry John Kponyo, Kwasi Adu-Boahen Opare, Ahmed Abdul-Rahman, Justice Owusu Agyemang. An Intelligent Irrigation System for Rural Agriculture[J]. International Journal of Applied Agricultural Sciences,2019,5(3).

3. Willians Ribeiro Mendes, Fábio Meneghetti U. Araújo, Ritaban Dutta,Derek M. Heeren. Fuzzy control system for variable rate irrigation using remote sensing[J]. Expert Systems With Applications,2019,124.

4. Jinmao Li,Hu He. Design of rice intelligent water-saving irrigation system based on agricultural internet of things[J]. Journal of Physics: Conference Series,2019,1176(5).

5. Kirtan Jha, Aalap Doshi, Poojan Patel. INTELLIGENT IRRIGATION SYSTEM USING ARTIFICIAL INTELLIGENCE AND MACHINE LEARNING: A COMPREHENSIVE REVIEW.[J]. International Journal of Advanced Research (IJAR),2018,6(10). 
6. Bertha Mazon-Olivo, Dixys Hernández-Rojas, José Maza-Salinas, Alberto Pan. Rules engine and complex event processor in the context of internet of things for precision agriculture[J]. Computers and Electronics in Agriculture,2018,154.

7. Jian Zhai Wu, Shuqing Han, Jifang Liu. Application Progress of Agricultural Internet of Things in Major Countries[J]. Journal of Physics: Conference Series,2018,1087(3).

8. Zhigang Liu, Qinchao Xu. An Automatic Irrigation Control System for Soilless Culture of Lettuce[J]. Water,2018,10(11). 